\title{
Adaptação transcultural e reprodutibilidade do Measure of the Quality of the Enviroment em indivíduos com hemiparesia
}

\section{Cross-cultural adaptation and reproducibility of the Measure of the Quality of the Environment in individuals with hemiparesis}

\author{
Iza de Faria-Fortini ${ }^{1}$, Marluce Lopes Basílio ${ }^{2}$, Fernanda Sabine Nunes \\ Assumpção ${ }^{3}$, Luci Fuscaldi Teixeira-Salmela ${ }^{4}$
}

http://dx.doi.org/10.11606/issn.2238-6149.v27i1p42-51

Faria-Fortini I, Basílio ML, Assumpção FSN, Teixeira-Salmela LF. Adaptação transcultural e reprodutibilidade do Measure of the Quality of the Enviroment em indivíduos com hemiparesia. Rev Ter Ocup Univ São Paulo. 2016 jan.-abr.;27(1):42-51.

RESUMO: A funcionalidade sofre a influência de aspectos relacionados à condição de saúde, bem como de fatores ambientais. Dentre os questionários que mensuram o impacto de fatores ambientais na funcionalidade, o Measure of the Quality of the Environment (MQE) é comumente utilizado. O objetivo deste estudo foi adaptar transculturalmente o MQE para o português-Brasil e avaliar sua reprodutibilidade. Após adaptação transcultural, o MQE foi aplicado em 28 indivíduos pós-AVE, duas vezes, com intervalo de sete a 10 dias. Foram calculados os coeficientes Kappa ponderado e de correlação intraclasse (CCI) para avaliação da confiabilidade teste-reteste. Os limites de concordância foram verificados por meio do gráfico Bland-Altman. Foram também calculados o erro padrão de medida (EPM) e a diferença mínima detectável (DMD). Os resultados mostraram que $81 \%$ dos itens apresentaram confiabilidade moderada a quase perfeita, e que os escores totais de facilitador e barreira apresentaram alta confiabilidade (ICC $>0,71$ ). Não foram observados erros sistemáticos entre o teste e o reteste e os valores EPM foram aceitáveis, sendo possível detectar mudança real da percepção dos fatores ambientais ao longo do tempo. O MQE-Brasil apresentou potencial para utilização na prática clínica e em pesquisas científicas.

DESCRITORES: Acidente vascular cerebral; Inquéritos e questionários; Meio ambiente; Reprodutibilidade dos testes.
Faria-Fortini I, Basílio ML, Assumpção FSN, Teixeira-Salmela LF. Cross-cultural adaptation and reproducibility of the Measure of the Quality of the Environment in individuals with hemiparesis. Rev Ter Ocup Univ São Paulo. 2016 Jan.-Apr.;27(1):42-51.

ABSTRACT: Functionality is influenced by aspects related to a given health condition, as well as by environmental factors. Amongst the questionnaires, that measure the impact of environmental factors on functionality, the Measure of the Quality of the Environment (MQE) is commonly used. The aim of this study was to cross-culturally adapt the MQE into the Brazilian-Portuguese language and to assess its reproducibility. Following the cross-cultural adaptation process, the MQE was applied to 28 stroke survivors on two occasions, seven to 10 days apart. Weighted Kappa and intra-class correlation coefficients (ICCs) were calculated to assess test-retest reliability, whereas the limits of agreement were verified by the Bland-Altman plots. The standard error of the measurement (SEM) and the ability to detect real changes (smallest real difference- SRD) were also calculated. The results indicated that $81 \%$ of the items showed moderate to almost perfect reliability (ICC $>0.71$ ). Systematic errors were not observed between the test and re-test scores and the SEM values were acceptable, being possible to detect real change on perceptions of environmental factors over time. The MQE-Brazil has potential to be used within clinical and research contexts.

KEYWORDS: Stroke; Surveys and questionnaires; Environment; Reproducibility of results.

\footnotetext{
* Este trabalho é parte da tese de doutorado 'Preditores da restrição na participação social em hemiparéticos' de Iza de Faria-Fortini, Programa de Pós-Graduação em Ciências da Reabilitação - Universidade Federal de Minas Gerais; apresentação de pôster no X Congresso Brasileiro de Doenças Cerebrovasculares - AVC 2015, Belo Horizonte, 15-17 out. 2015. Fontes de auxílio: CAPES, CNPq e FAPEMIG 1. Universidade Federal de Minas Gerais (UFMG), Belo Horizonte, MG, Brasil. E-mail: izafaria@yahoo.com.br

2. Universidade Federal de Minas Gerais (UFMG), Belo Horizonte, MG, Brasil. E-mail: marlucelb@yahoo.com.br

3. Centro de Reabilitação Noroeste da Rede do Sistema Único de Saúde, Belo Horizonte, MG, Brasil. E-mail: fernandasabine@yahoo.com.br. 4. Universidade Federal de Minas Gerais (UFMG), Belo Horizonte, MG, Brasil. E-mail: 1fts@ufmg.br

Endereço para correspondência: Prof ${ }^{a}$ Luci Fuscaldi Teixeira-Salmela. Departamento de Fisioterapia, Universidade Federal de Minas Gerais - Campus Pampulha. Avenida Antônio Carlos, 6627. Belo Horizonte, MG, Brasil. CEP: 31270-901. E-mail: 1fts@ufmg.br
} 


\section{INTRODUÇÃO}

$\mathrm{S}$ egundo a Classificação Internacional de Funcionalidade, Incapacidade e Saúde (CIF), fatores ambientais compõem o ambiente físico, social e de atitude no qual as pessoas vivem e conduzem suas vidas ${ }^{1}$. A CIF apresenta uma lista abrangente desses fatores que incluem: produtos e tecnologias; ambiente natural e mudanças feitas pelo homem; apoio e relacionamentos; atitudes; e serviços, sistemas e políticas ${ }^{1}$. Esses fatores são externos ao indivíduo e interagem com os componentes de Estruturas e Funções do Corpo, Atividade e Participação, atuando como facilitadores ou barreiras no processo de funcionalidade e incapacidade relacionado a diversas condições de saúde ${ }^{1}$.

O acidente vascular encefálico (AVE) é uma das principais causas de incapacidade crônica entre adultos no Brasil ${ }^{2}$. Após o AVE, é comum a ocorrência de limitações na realização de atividades cotidianas e restrição na participação social ${ }^{3}$. O conhecimento acerca da influência dos fatores ambientais na emergência desses aspectos negativos pode orientar modificações de ambientes para atender às necessidades desses indivíduos, uma vez que deficiências e incapacidades de longo prazo ainda são difíceis de recuperar apesar dos avanços tecnológicos ${ }^{4}$. Nesse sentido, torna-se necessária a utilização de instrumentos padronizados para mensuração do impacto dos fatores ambientais para a elaboração de estratégias que visem o aumento da funcionalidade de indivíduos pós-AVE por meio da redução das barreiras e aumento dos facilitadores ambientais.

O Measure of the Quality of the Environment (MQE) é um questionário que mensura a percepção do indivíduo sobre o ambiente físico e social, isto é, se cada fator ambiental é percebido como facilitador ou barreira durante o desempenho de atividades diárias e papéis sociais $^{5}$. O MQE contempla seis domínios, que abrangem os cinco capítulos do componente Fatores Ambientais da CIF $^{5,6}$ : atitudes e suporte social, trabalho e renda, serviços governamentais e públicos, igualdade de oportunidade e orientações políticas, referentes ao ambiente social; e estrutura física e acessibilidade e tecnologia, referentes ao ambiente físico. Há versões longa (84 itens) e reduzida (26 itens) do MQE. Estudo prévio, com adultos com paralisia cerebral, indicou que $85 \%$ dos itens da versão longa do MQE obtiveram confiabilidade em torno de $60 \%{ }^{7}$. No presente estudo, foi utilizada a versão reduzida, que mantém os domínios contemplados na versão longa, uma vez que o uso de questionários abreviados envolve menor tempo para aplicação, cálculo e interpretação do escore, o que pode facilitar a utilização na prática clínica e em pesquisas ${ }^{8}$.

O MQE foi desenvolvido nas línguas inglesa e francesa, sendo necessária a adaptação transcultural para a sua utilização na população brasileira ${ }^{9,10}$. Após esse processo, a avaliação das propriedades de medida é recomendada para confirmar se o questionário é adequado para a aplicação pretendida ${ }^{9,10}$. Uma propriedade de medida considerada como requisito essencial para todas as medidas de desfecho é a reprodutibilidade, que diz respeito ao grau no qual medidas repetidas, em indivíduos estáveis, fornecem resultados semelhantes ${ }^{11}$. Desta forma, os objetivos deste estudo foram realizar a adaptação transcultural do MQE para a língua portuguesaBrasil e avaliar a sua reprodutibilidade (confiabilidade e concordância) em indivíduos com hemiparesia crônica.

\section{MÉTODO}

Este estudo metodológico foi desenvolvido em duas etapas: adaptação transcultural e avaliação da reprodutibilidade do MQE. O desenvolvimento da versão brasileira do MQE foi autorizado pela International Network on the Disability Creation Process (INDCP), portadora dos direitos autorais.

\section{Participantes}

Foram recrutados indivíduos com hemiparesia residentes na comunidade. Os critérios de inclusão foram: diagnóstico clínico de AVE primário ou recorrente com tempo de evolução acima de seis meses; idade igual ou superior a 20 anos; hemiparesia, caracterizada pelo aumento de tônus dos músculos flexores de cotovelo e/ou pela fraqueza muscular de preensão manual ou extensores de joelho, determinada por uma diferença superior a $10 \%$ e 15\%, respectivamente, entre o lado parético e o não parético ${ }^{12}$. Foram excluídos os indivíduos com déficits cognitivos avaliados pelo Mini-Exame do Estado Mental (ponto de corte 13 para analfabetos, 18 para baixa e média escolaridade e 26 para alta escolaridade $)^{13}$; dificuldade de expressão verbal; hemiparesia dupla e outras condições musculoesqueléticas ou neurológicas incapacitantes. Segundo sugestão de Hobart et al..$^{14}$, é necessária uma amostra de no mínimo 20 participantes para avaliação de reprodutibilidade.

\section{Descrição do MQE}

O MQE é um questionário aplicado por meio de entrevista, durante a qual os indivíduos são solicitados 
a estimar a influência de fatores ambientais durante a execução de atividades cotidianas como: facilitadores, quando os fatores ambientais auxiliam na realização de tarefas diárias; barreiras, quando os fatores ambientais dificultam a realização de tarefas diárias; ou sem influência, quando os fatores ambientais não interferem na realização de tarefas diárias ${ }^{5}$. Para pontuar a influência de cada um dos 26 fatores ambientais, é utilizada uma escala Likert de sete pontos, variando de -3 (obstáculo maior) a 3 (facilitador maior) $)^{5}$. O escore zero indica que o item não é percebido nem como facilitador ou como barreira, não exercendo influência na funcionalidade 5 . Dois escores finais contínuos são calculados: obstáculo ambiental, que corresponde à média de todas as respostas negativas $(-1$, obstáculo menor; -2, obstáculo médio; -3, obstáculo maior) e facilitador ambiental, que equivale à média de todas as respostas positivas $(+1$, facilitador menor; +2 , facilitador médio; +3 , facilitador maior $)^{5}$. Estes escores proporcionam informações sobre a magnitude de barreiras e/ou facilitadores na realização de atividades e na participação.

\section{Procedimento}

\section{Adaptação transcultural}

Segundo recomendações de Beaton et al. ${ }^{9}$, o MQE foi traduzido para o português-Brasil do ponto de vista semântico, cultural e conceitual por dois tradutores bilíngues, cujo primeiro idioma era o português, com formação acadêmica diferente, sendo um da área da saúde (etapa I). A etapa II consistiu da síntese das versões traduzidas, gerando uma versão-consenso. A partir desta versão, foi realizada a retrotradução, etapa III, de forma independente por dois tradutores bilíngues independentes, cuja língua-mãe era o inglês, com formação acadêmica distinta, sendo um deles da área da saúde. Estes não tiveram acesso ao questionário original e não estavam cientes dos objetivos do estudo. A etapa IV correspondeu à análise do comitê de especialistas, composto pela primeira autora, três fisioterapeutas, um tradutor e um retrotradutor. Esse comitê analisou a clareza, pertinência e equivalência entre as versões traduzidas, retrotraduzidas e a original, consolidou essas versões e desenvolveu a versão pré-final. Por sugestão dos autores, foram acrescidas observações e exemplos no corpo do questionário para ampliar a possibilidade de compreensão das questões.

Para verificação da compreensão dos itens (etapa V), a versão pré-final foi aplicada em 10 indivíduos com hemiparesia com média de idade de $59 \pm 9$ anos e tempo de evolução de $8 \pm 5$ anos. Durante a aplicação, a cada item do
MQE foi acrescida uma pergunta referente à compreensão do mesmo, baseado numa escala dicotômica (fácil ou difícil). Não foram observados dúvidas ou conflitos de terminologia, sendo, desta forma, considerada satisfatória a equivalência semântica e cultural. $\mathrm{O}$ processo de adaptação transcultural (etapa VI) foi concluído, sendo o questionário denominado MQE-Brasil (Tabela 1).

\section{Avaliação da reprodutibilidade}

Inicialmente, os participantes foram informados sobre o objetivo e procedimentos do estudo e assinaram o termo de consentimento livre e esclarecido. Em seguida, foram coletados dados demográficos e clínicos para caracterização da amostra. O comprometimento motor foi mensurado pela escala de Fugl Meyer (EFM) que destina um total de 100 pontos para a função motora normal, sendo que escore inferior a 50 indica comprometimento motor grave; entre 51 a 84 comprometimento motor marcante; entre 85 a 95 comprometimento motor moderado; enquanto escores entre 96 e 99 representam comprometimento motor leve ${ }^{15}$. O MQE-Brasil foi aplicado duas vezes, com intervalo de sete a 10 dias, pelo mesmo examinador devidamente treinado, que seguiu instruções propostas pelo manual ${ }^{5}$.

\section{Análise estatística}

Estatísticas descritivas foram utilizadas para caracterização da amostra. Para análise da reprodutibilidade, foi avaliada a confiabilidade teste-reteste e a concordância. $\mathrm{Na}$ análise da confiabilidade teste-reteste, foram avaliados a confiabilidade dos itens e do escore total. A confiabilidade teste-reteste de cada item foi realizada por meio do cálculo do coeficiente Kappa com ponderação quadrática $\left(\kappa_{\mathrm{w}}\right)$, a fim de diferenciar a magnitude das discrepâncias entre as respostas $^{16}$. A interpretação do Kappa ponderado, cujo escore varia de 0 (ausência de confiabilidade) a 1,0 (confiabilidade perfeita), foi realizada conforme proposto por Landis e $\operatorname{Koch}^{17}(0,00>\kappa<0,20$, fraca; $0,20>\kappa<0,40$, razoável; $0,40>\kappa<0,60$, moderada; $0,60>\kappa<0,80$, boa; e $\kappa>0,80$ quase perfeita). A confiabilidade teste-reteste dos escores totais (barreira e facilitador) foi determinada pela análise do coeficiente de correlação intraclasse $(\mathrm{CCI})^{16}$. $\mathrm{O}$ CCI é calculado a partir da relação entre a variância entre sujeitos e a variância total, com valores que variam de 0 (ausência de confiabilidade) a 1,0 (confiabilidade perfeita) ${ }^{16}$. Os critérios propostos por Munro $^{18}$ foram adotados para a interpretação do grau de confiabilidade: muito baixa: 0 a 0,25 ; baixa: 0,26 a 0,49 ; moderada: 0,50 a 0,69 ; alta: 0,70 a 0,89 ; muito alta: 0,90 a 1,00 . 
Tabela 1 - Versão final traduzida do MQE-Brasil

Levando em consideração suas habilidades e limitações pessoais, indique o quanto as situações ou fatores geralmente influenciam sua vida diária

\begin{tabular}{|c|c|c|c|c|c|c|c|c|}
\hline \multicolumn{3}{|c|}{ Obstáculo } & \multirow{2}{*}{ 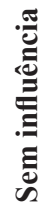 } & \multicolumn{3}{|c|}{ Facilitador } & \multirow{2}{*}{ 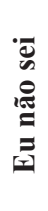 } & \multirow{2}{*}{ 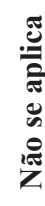 } \\
\hline$\stackrel{\bar{\sigma}}{\frac{\dot{\pi}}{\Sigma}}$ & 胥 & 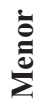 & & 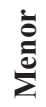 & : & $\frac{\bar{\Xi}}{\bar{\pi}}$ & & \\
\hline-3 & -2 & -1 & $\mathbf{0}$ & +1 & +2 & +3 & & \\
\hline
\end{tabular}

\section{Item}

1- Apoio daqueles a sua volta (família, amigos, colegas)

2- As atitudes/comportamentos daqueles a sua volta (família, amigos, colegas)

3- A disponibilidade/oferta atual de empregos na sua comunidade

4- As características do seu ambiente de trabalho (estrutura física do local de trabalho, carga horária)

5- Sua renda pessoal

Obs: renda pessoal inclui salário, pensão, aposentadoria e outros rendimentos

6- Seguros e outros programas de compensação financeira (plano de saúde, seguro de vida, benefícios sociais)

7- Lojas e serviços em sua comunidade

8- Serviços de atendimento domiciliar (saúde, faxina, reabilitação, serviços de entrega à domicílio)

9- Serviços educacionais (escolas, cursos profissionalizantes, faculdades/ universidades)

10- O veículo pessoal que você usa

11- Serviços de transporte público

12- Rádio e televisão (acesso, qualidade da informação, legenda)

13- Comunicação eletrônica (telefone, fax, e-mail, internet)

14- Serviços comunitários e culturais em sua comunidade (organizações culturais, esportivas e religiosas)

Obs: inclui cinema, teatro, biblioteca, missa, grupo de autoajuda, etc.

15- Acesso físico de sua residência

16- Acesso físico de prédios em sua comunidade que você precisa entrar (banco, correio, prefeitura, posto de saúde)

17- Acesso de ruas em sua comunidade (ruas, calçadas, meio fio, cruzamentos)

18- Condições climáticas (frio, calor, chuva, umidade)

19- Intensidade da luz

20- Intensidade do barulho ou som

21- Tempo permitido para executar tarefas (tempo necessário para vestir-se, ir ao trabalho, etc.)

22- Objetos que você usa (itens de trabalho ou estudo, móveis, decoração, eletrodomésticos, equipamento eletrônico)

23- Dispositivos de auxílio e adaptações, por exemplo, óculos, bengala e corrimão (disponibilidade, uso, manutenção)

24- Participação em decisões em sua comunidade (assembleia/reunião pública, eleições)

25- Procedimentos governamentais e administrativos (documentos e formulários necessários para a solicitação de serviços bancários, renovações de aposentadoria, carteira de motorista e benefícios)

26- Procedimentos administrativos e regras (regras para fumantes, regras em estacionamentos e normas burocráticas) 
Faria-Fortini I, et al. Adaptação transcultural e reprodutibilidade. Rev Ter Ocup Univ São Paulo. 2016 jan./abr.;27(1):42-51.

Para avaliação da concordância entre os escores obtidos no teste-reteste, foi realizada análise do gráfico Bland-Altman, bem como cálculo do erro padrão de medida (EPM) e da diferença mínima detectável (DMD). O gráfico Bland-Altman analisa os limites de concordância ou discordância entre as duas medidas (teste-reteste), sendo a média da diferença entre as duas medidas (teste 2 - teste 1) calculada, bem como seu intervalo de confiança de $95 \%$, para determinar a magnitude da discordância e a existência de erros e padrões sistemáticos entre as duas ocasiões de teste ${ }^{16}$.

O EPM é uma estimativa da variabilidade entre medidas após a realização de medidas repetidas, sendo calculado pela equação $[\mathrm{EPM}=\mathrm{DP} \times \sqrt{ }(1-\mathrm{CCI})]$, onde DP é o desvio padrão encontrado na primeira aplicação ${ }^{11,19}$. A DMD consiste no valor da mudança no escore que é maior do que o erro de medição, sendo desta forma uma medida importante ao analisar a mudança real no desempenho dos indivíduos após repetidas medições em um teste ${ }^{11,19}$. A DMD foi calculada pela fórmula $[\mathrm{DMD}=1,96 \times \mathrm{EPM}$ x $\sqrt{ }(2)]^{11,19}$. Para o EPM, foi calculado o EPM\% que corresponde a porcentagem do EPM relacionado ao escore total, sendo interpretado da seguinte forma: $\leq 5 \%$ muito bom; $>5 \%$ e $\leq 10 \%$ bom; $>10 \%$ e $\leq 20 \%$ duvidoso e $>$ $20 \%$ negativo $^{20}$. Como a DMD é baseada no EPM, não foi definido nenhum critério para sua análise ${ }^{20}$.

Todas análises foram realizadas pelo Statistical Package for Social Sciences (SPSS ${ }^{\circledR}$ ), versão 15.0, e pelo site VassarStats com um nível de significância de 5\%.

\section{Aspectos éticos}

O estudo foi aprovado pelo Comitê de Ética em Pesquisa da Universidade Federal de Minas Gerais sob o $n^{\circ}$ CAAE 06609312.0.0000.5149.

\section{RESULTADOS}

\section{Caracterização da amostra}

Dos 115 indivíduos que compareceram à primeira avaliação, apenas 28 compareceram para a segunda administração do questionário. Os 28 participantes tinham em média $61 \pm 10$ anos de idade (38 a 83 anos), sendo que $57 \%$ do total da amostra eram homens. Dentre os participantes, $57,1 \% \quad(n=16)$ não concluíram o ensino fundamental; $10,7 \%(\mathrm{n}=3)$ concluíram o ensino fundamental; 10,7\% $(n=3)$ concluíram o ensino médio; $10,7 \%(n=3)$ concluíram o ensino superior; 7,2\% $(n=2)$ eram analfabetos e 3,6\% $(\mathrm{n}=1)$ possuíam o ensino médio incompleto. A média de tempo de evolução foi de $5 \pm 5$ anos. Com relação ao comprometimento motor, 25,0\% $\quad(n=7)$ apresentaram comprometimento motor moderado; $21,3 \% \quad(n=6)$ comprometimento leve; $17,9 \% \quad(n=5)$ comprometimento grave; $17,9 \%(n=5)$ comprometimento marcante e $17,9 \%$ $(n=5)$ não apresentaram alteração da função motora.

\section{Confiabilidade teste-reteste}

Os coeficientes Kappa estão reportados na Tabela 2. Dos 26 itens do MQE-Brasil, cinco (19\%) apresentaram confiabilidade quase perfeita $\left(\kappa_{\mathrm{w}} 0,83-0,95\right)$; oito $(31 \%)$ confiabilidade boa $\left(\kappa_{\mathrm{w}} 0,61-0,75\right)$; oito $(31 \%)$ confiabilidade moderada $\left(\kappa_{w} 0,40-0,59\right)$; e, em cinco $(19 \%)$ confiabilidade razoável $\left(\kappa_{\mathrm{w}} 0,21-0,39\right)$.

Os gráficos de concordância de Bland-Altman são apresentados na Figura 1. Para o escore de facilitador (Figura 1A), observa-se a média das diferenças 0,06 $\pm 0,42$ (IC 95\%: -0,76 a 0,89); para o escore de barreira (Figura 1B), observa-se a média das diferenças $-0,23 \pm 0,81$ (IC 95\%: -1,83 a 1,36). Em ambos os casos, a média das diferenças estão próximas de zero, indicando que os escores provenientes da primeira e da segunda aplicação do MQE foram similares, não sendo encontrado um padrão na distribuição que indicasse a ocorrência de erro sistemático.

Tabela 2 - Classificação dos coeficientes Kappa da análise de confiabilidade teste-reteste por questão do MQE-Brasil (n=28)

\begin{tabular}{l|l}
\hline $\begin{array}{l}\text { Parâmetros de referência para } \\
\text { o coeficiente de confiabilidade } \\
\text { Kappa }\end{array}$ & Questões \\
\hline$>0,80$ (quase perfeita) & $3,4,6,10,17$ \\
$0,60-0,80$ (boa) & $1,2,5,9,11,13,14,18$ \\
$0,40-0,60$ (moderada) & $7,12,16,19,20,22,24$, \\
$0,20-0,40$ (razoável) & 25 \\
\hline
\end{tabular}

Como podem ser observados nas Figuras 1A e 1B, dois participantes apresentaram maiores diferenças entre as duas aplicações, tanto nos escores de facilitador, quanto de barreira. Sendo assim, o CCI, EPM, EPM\%, DMD e $\mathrm{DMD} \%$ foram calculados com a inclusão dos indivíduos com comportamento atípico $(n=28)$ e sem os indivíduos com comportamento atípico $(n=26)$ (Tabela 3). Os CCI foram altos em ambas as situações, tanto para o escore facilitador, quanto para o escore barreira. Os valores de EPM\% foram considerados bons (abaixo de 10\%) para o escore facilitador com amostra completa e com a exclusão dos dois indivíduos com comportamento atípico. Para o escore barreira, EPM\% foi aceitável apenas com a exclusão dos dois indivíduos com comportamento atípico. 

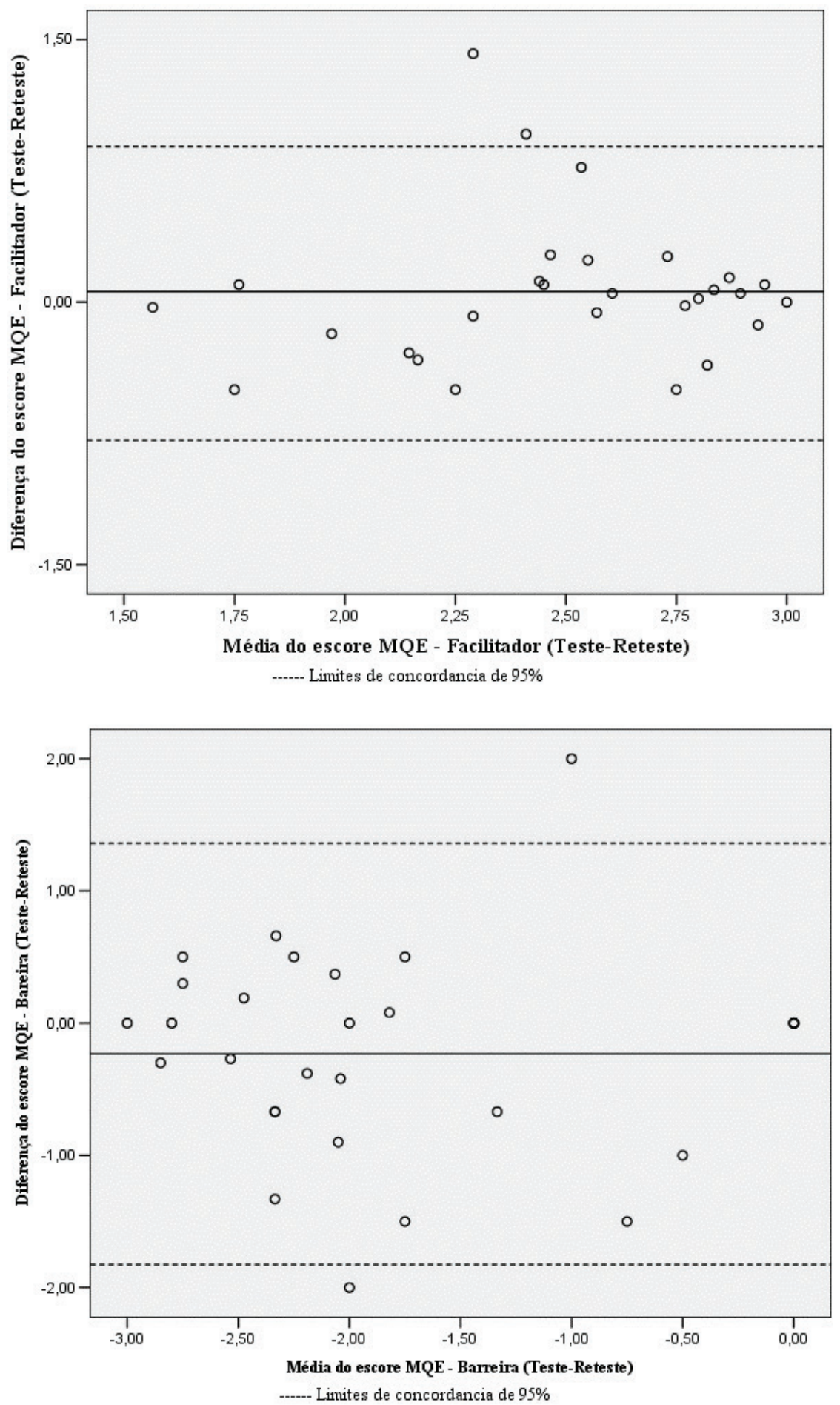

$1 \mathrm{~A}$

Figura 1 - Gráfico de concordância Bland-Altman dos escores teste-reteste do MQE - Brasil $(n=28)$. No eixo das abscissas $(X)$, encontram-se as médias dos escores entre o teste e o reteste, e no eixo das ordenadas (y), há a indicação da diferença entre os escores da primeira (teste) e da segunda (reteste) aplicação do MQE-Brasil 
Faria-Fortini I, et al. Adaptação transcultural e reprodutibilidade. Rev Ter Ocup Univ São Paulo. 2016 jan./abr.;27(1):42-51.

Tabela 3 - Reprodutibilidade do MQE

\begin{tabular}{l|c|c}
\hline & $\mathbf{n}=\mathbf{2 8}$ & $\mathbf{n}=\mathbf{2 6}$ \\
\hline Facilitador & \multicolumn{3}{|c}{} \\
\hline CCI (IC 95\%) & $0,71(0,37-0,86)$ & $0,88(0,73-0,94)$ \\
\hline EPM & 0,24 & 0,16 \\
\hline EPM\% & 8 & 5 \\
\hline DMD & 0,66 & 0,44 \\
\hline DMD\% & 22 & 14 \\
\hline Barreira & \multicolumn{2}{|}{} \\
\hline CCI (IC 95\%) & $0,79(0,55-0,90)$ & $0,88(0,74-0,95)$ \\
\hline EPM & 0,43 & 0,31 \\
\hline EPM\% & 14 & 10 \\
\hline DMD & 1,19 & 0,85 \\
\hline DMD $\%$ & 40 & 28 \\
\hline
\end{tabular}

$\mathrm{CCI}=$ Coeficiente de confiabilidade intraclasse; $\mathrm{IC}=$ intervalo de confiança; $\mathrm{EPM}=$ erro padrão da medida; $\mathrm{DMD}=$ diferença mínima detectável

\section{DISCUSSÃO}

Este estudo realizou a adaptação transcultural para o Brasil de um questionário de avaliação de fatores ambientais, cujos conceitos são coerentes com o referencial teórico da CIF. A avaliação da reprodutibilidade evidenciou que o MQE-Brasil apresentou confiabilidade teste-reteste adequada e valores de concordância dentro dos limites aceitáveis. Estes resultados indicam o potencial para sua aplicação na prática clínica e em pesquisas científicas, com o objetivo de avaliar a influência de fatores ambientais na funcionalidade de indivíduos com hemiparesia. A primeira etapa, adaptação transcultural, seguiu diretrizes propostas ${ }^{9,10}$. Por sugestão dos autores do $\mathrm{MQE}$, não foram alterados ou suprimidos itens com divergência cultural. Desta forma, somente foram adicionados ao texto original observações e exemplos que ampliaram a possibilidade de compreensão.

Dentre os métodos de avaliação de confiabilidade teste-reteste, optou-se pelo coeficiente Kappa ponderado para análise da confiabilidade dos itens por ser um método robusto, consistente com o delineamento do presente estudo e adequado para avaliar questionários com variáveis categóricas e resultados expressos em mais de duas categorias ${ }^{16}$. $\mathrm{Na}$ análise do Kappa ponderado, $81 \%$ dos itens apresentaram confiabilidade moderada a quase perfeita, com valor superior ao reportado por Boschen et al.?. No entanto, apesar de aceitáveis, valores de coeficientes razoáveis foram encontrados para os itens serviços de atendimento domiciliar (questão 8), acesso físico da residência (questão 15), tempo permitido para executar tarefas (questão 21), dispositivos auxiliares e adaptações (questão 23) e procedimentos administrativos e regras (questão 26). Deve-se considerar que os coeficientes de confiabilidade estão relacionados com a estabilidade de medidas repetidas, sendo vulneráveis à influência de alguns fatores, tais como fatores pessoais ou referentes ao próprio questionário ${ }^{11}$. As alterações de humor estão dentre os fatores pessoais que podem ter interferido na estabilidade entre as medições ${ }^{11}$. Além disso, a descrição breve dos itens do MQE e a relativa baixa escolaridade de grande parte da amostra podem ter ocasionado dificuldades por parte dos participantes em considerarem a influência desses fatores ambientais na execução de suas atividades cotidianas $^{21}$.

Dois indivíduos na análise dos facilitadores e barreiras ambientais apresentaram comportamento atípico, com maiores diferenças entre os escores na primeira e segunda avaliação. Quando analisada a amostra completa ( $\mathrm{n}=28)$, não foi observada presença de erro sistemático, ou seja, não houve tendência de subestimar ou superestimar os facilitadores e barreiras ambientais na reaplicação do questionário. Este fato pode ser visualizado nos gráficos de Bland-Altman, uma vez que os pontos foram distribuídos aleatoriamente em torno do zero.

A análise de confiabilidade teste-reteste dos escores totais foi realizada com e sem os indivíduos com comportamento atípico. A confiabilidade dos escores de facilitador e barreira, analisada por meio do CCI, foi alta em ambas as situações, indicando consistência do MQE ao avaliar o impacto de fatores ambientais na funcionalidade. Embora o CCI seja um dos métodos mais comuns de avaliação da confiabilidade, devem ser realizadas análises complementares, uma vez que o CCI não é suficiente para uma avaliação abrangente da reprodutibilidade de uma medida ${ }^{16}$. O CCI é utilizado para avaliar a consistência entre escores provenientes de aplicações repetidas, considerando apenas a variação da amostra ${ }^{16}$, sendo então requerido para avaliar se o questionário pode ser usado para fins discriminativos, quando se quer realizar a distinção entre os indivíduos ${ }^{11}$. Porém, para avaliar se o questionário pode ser usado para fins avaliativos, ou seja, quando o objetivo é medir a mudança do estado de saúde de um indivíduo ao longo do tempo, por exemplo, antes e após uma intervenção, a detecção da variação individual entre duas ocasiões de teste, analisada por meio do cálculo de EPM e DMD, é necessária ${ }^{11}$.

O cálculo de EPM, EPM\%, DMD e DMD\% também foi realizado com e sem os indivíduos com comportamento atípico. Após a retirada de indivíduos com comportamento atípico, observou-se que os valores do EPM (\%) encontraram-se dentro dos limites considerados como bons (abaixo de $10 \%)^{20}$, indicando que os escores 
foram estáveis e tiveram baixa variabilidade. Em termos clínicos, para qualquer escore observado, o EPM quantifica a amplitude sobre a qual é esperada a variação do escore devido ao erro da medida, sendo que esta informação deve ser considerada nas decisões clínicas ${ }^{22}$. Desta forma, após a aplicação do questionário em um mesmo indivíduo, em momentos distintos, uma variação de 0,16 e 0,31 para os escores de facilitador e barreira, respectivamente, está relacionada ao erro da medida e não a uma real alteração dos fatores ambientais. Por exemplo, para um indivíduo com escore $+2,0$ (facilitador), pode ser esperado, em uma aplicação subsequente, um escore variando entre $+1,84$ a $+2,16(+2,0 \pm 0,16)$ em decorrência do erro da medida. Similarmente, se o escore para barreira for $-2,0$, é esperada uma variação entre -2,31 e -1,69 $(-2,0 \pm 0,31)$. Adicionalmente a essa interpretação do EPM, o DMD pode facilitar a compreensão de resultados provenientes da aplicação do MQE. Os valores de DMD encontrados indicam que alterações na percepção de fatores ambientais ao longo do tempo maiores do que 0,44 e 0,85 para os escores: facilitador e barreira, respectivamente, indicam mudanças reais acima do erro da medida ${ }^{22}$. A comparação dos índices de confiabilidade e concordância da resposta encontrados neste estudo com estudos prévios é limitada, uma vez que este foi o primeiro estudo a utilizar o CCI para análise da confiabilidade teste-reteste e determinar o EPM e a DMD do MQE.

Neste estudo, dados provenientes de 28 indivíduos foram analisados para avaliar a reprodutibilidade do MQE. Não há um consenso quanto o tamanho da amostra em estudos de confiabilidade, embora recentes publicações proporcionem sugestões para auxiliar no cálculo amostral ${ }^{14,23}$. Esse cálculo é importante porque a inadequação do tamanho da amostra pode acarretar uma subestimação dos valores de confiabilidade e uma superestimação dos valores de erro da medida ${ }^{24}$. Hobart et al. ${ }^{14}$ analisaram a influência do tamanho da amostra na estabilidade das estimativas de confiabilidade, incluindo teste-reteste, e nas inferências feitas a partir dessas estimativas, sugerindo que uma amostra mínima de 20 indivíduos fornece estimativas estáveis em magnitude e interpretação. No entanto, segundo recomendações do Consensus-based standards for the selection of health measurement instruments $(\mathrm{COSMIN})^{23}$, o tamanho mínimo da amostra para avaliação da reprodutibilidade de um questionário deve ser de 30 participantes. Ressalta-se que a definição do tamanho da amostra deve também considerar fatores como aporte financeiro e disponibilidade dos voluntários ${ }^{25}$. No caso desse estudo, dentre os 115 voluntários que participaram da primeira avaliação, somente 28 indivíduos aceitaram participar da segunda aplicação do MQE, evidenciando as dificuldades para a realização desse tipo de estudo.

O MQE-Brasil é um questionário de rápida aplicação (em torno de 20 minutos) e fácil interpretação dos escores. Entretanto, como na análise individual dos itens pelo coeficiente Kappa, algumas questões apresentaram concordância razoável, sugere-se que o entrevistador reforce as instruções iniciais e enfatize os exemplos, especialmente para aqueles itens que apresentaram variabilidade nas respostas, de forma a assegurar que o escore represente a percepção real do indivíduo sobre a influência de facilitadores e barreiras na funcionalidade. Vale ressaltar que os resultados deste estudo podem ser generalizados para indivíduos com características similares as da amostra.

Em conclusão, o MQE-Brasil apresentou reprodutibilidade adequada para ser utilizado com propósitos discriminativo e avaliativo, com potencial para utilização na prática clínica e em pesquisas científicas indivíduos com hemiparesia crônica.

Autoria e indicação de responsabilidade: I. Faria-Fortini, M.L. Basílio, F.S.N. Assumpção - contribuíram com a concepção, o planejamento, a organização e o desenvolvimento do projeto; participaram de todo o seu desenvolvimento, desde a coleta dos dados e interpretação dos resultados até a elaboração do manuscrito, revisão crítica do seu conteúdo e aprovação da sua versão final. L.F. Teixeira-Salmela colaborou com o planejamento e desenvolvimento do projeto e participou das etapas de tabulação dos dados, cálculos estatísticos e interpretação dos resultados, elaboração do manuscrito e revisão crítica do seu conteúdo e aprovação da sua versão final. 
Faria-Fortini I, et al. Adaptação transcultural e reprodutibilidade. Rev Ter Ocup Univ São Paulo. 2016 jan./abr.;27(1):42-51.

\section{REFERENNCIAS}

1. Organização Mundial de Saúde, Organização Panamericana de Saúde. CIF- Classificação Internacional de Funcionalidade, Incapacidade e Saúde. São Paulo: Edusp; 2003.

2. Brasil. Ministério da Saúde. Secretaria de Atenção à Saúde. Departamento de Ações Programáticas Estratégicas. Diretrizes de atenção à reabilitação da pessoa com acidente vascular cerebral. Brasília: Ministério da Saúde; 2013.

3. Meneses KVP, Duarte JS, Alencar VO, Pereira ACS. Perfil sócio demográfico e áreas de desempenho ocupacional afetadas em pacientes pós-AVE atendidos por um serviço de terapia ocupacional. Rev Ter Ocup Univ São Paulo. 2012;23(2):107-12. doi: http://dx.doi.org/10.11606/issn.2238-6149.v23i2p107-112.

4. Langhorne P, Bernhardt J, Kwakkel G. Stroke rehabilitation. Lancet. 2011;377(9778):1693-702. doi: http://dx.doi. org/10.1016/S0140-6736(11)60325-5.

5. Fougeyrollas P, Noreau L, St-Michel G, Boschen K. Measure of the quality of the environment - short version. Québec: International Network of the Disability Creation Process; 2008.

6. Alvarelhão J, Silva A, Martins A, Queirós A, Amaro A, Rocha N, Lains J. Comparing the content of instruments assessing environmental factors using the International Classification of Functioning, Disability and Health. J Rehabil Med. 2012;44:1-6. doi: 10.2340/165019770905 .

7. Boschen K, Noreau L, Fougeyrollas P. A new instrument to measure the quality of environment for persons with physical disabilities. Arch Phys Med Rehabil. 1998;79(10):1331.

8. Campbell A, Hemsley S. Outcome Rating Scale and Session Rating Scale in psychological practice: Clinical utility of ultra-brief measures. Clin Psychol. 2009;13(1):1-9. doi: 10.1080/13284200802676391.

9. Beaton DE, Bombardier C, Guillemin F, Ferraz MB. Guidelines for the process of cross-cultural adaptation of self-report measures. Spine. 2000;25(24):3186-91.

10. Costner WJ, Mancini MC. Recomendações para a tradução e adaptação transcultural de instrumentos para a pesquisa e a prática em terapia ocupacional. Rev Ter Ocup Univ São Paulo. 2015;26(1):50-7. doi: http://dx.doi.org/10.11606/ issn.2238-6149.v26i1p50-57.

11. De Vet HCW, Terwee CB, Knol DL, Bouter LM. When to use agreement versus reliability measures. J Clin Epidemiol. 2006;59(10):1033-9. doi: 10.1016/j. jclinepi.2005.10.015.

12. Faria-Fortini I, Michaelsen SM, Cassiano JG, TeixeiraSalmela LF. Upper extremity function in stroke subjects: relationships between the International Classification of
Functioning, Disability and Health domains. J Hand Ther. 2011;24(3):257-64. doi: 10.1016/j.jht.2011.01.002.

13. Bertolucci PH, Brucki SMD, Campacci SR, Juliano Y. O mini-exame do estado mental em uma população geral: impacto da escolaridade. Arq Neuro-Psiquiatr. 1994;52(1):1-7. doi: http://dx.doi.org/10.1590/S0004282X1994000100001.

14. Hobart JC, Cano SJ, Warner TT, Thompson AJ. What sample sizes for reliability and validity studies in neurology? J Neurol. 2012;259(12):2681-94. doi: 10.1007/s00415-0126570-y.

15. Fugl-Meyer AR. Post-stroke hemiplegia assessment of physical properties. Scan J Rehabil Med Suppl. 1980;7(7):85-93.

16. Portney LG, Watkins MP. Foundations of clinical research: applications to practice. 3rd ed. New Jersey: Prentice Hall; 2008.

17. Landis JR, Koch GG. The measurement of observer agreement for categorical data. Biometrics. 1977;33(1):15974. doi: $10.2307 / 2529310$.

18. Munro BH. Statistical methods for health care research. 5th ed. Philadelphia: Lippincott, Williams \& Wilkins; 2005.

19. Pinheiro MB, Scianni AA, Ada L, Faria CD, TeixeiraSalmela LF. Reference values and psychometric properties of the Lower Extremity Motor Coordination Test. Arch Phys Med Rehabil. 2014;95(8):1490-7. doi: 10.1016/j. apmr.2014.03.006.

20. Ostelo RWJG, de Vet HCW, Knol DL, van den Brandt PA. 24-item Roland-Morris Disability Questionnaire was preferred out of six functional status questionnaires for postlumbar disc surgery. J Clin Epidemiol. 2004;57(3):268-76. doi: 10.1016/j.jclinepi.2003.09.005.

21. Furtado SRC, Sampaio RF, Vaz DV, Pinho BAS, Nascimento IO, Mancini MC. Versão brasileira do instrumento de avaliação ambiental Craig Hospital Inventory of Environmental Factors (CHIEF): tradução, adaptação cultural e confiabilidade. Braz J Phys Ther. 2014;18(3):259-67. doi: http://dx.doi.org/10.1590/bjptrbf.2014.0036.

22. Overend TJ, Wuori-Fearn JL, Kramer JF, Macdermid JC. Reliability of a patient-rated forearm evaluation questionnaire for patients with lateral epicondylitis. $J$ Hand Ther. 1999;12(1):31-7. doi: 10.1016/S0894-1130(99)80031-3.

23. Terwee CB, Mokkink LB, Knol DL, Ostelo RWJG, Bouter LM, De Vet HCW. Rating the methodological quality in systematic reviews of studies on measurement properties: a scoring system for the COSMIN checklist. Qual Life Res. 2012;21(4):651-7. doi: 10.1007/s11136-011-9960-1. 
Faria-Fortini I, et al. Adaptação transcultural e reprodutibilidade. Rev Ter Ocup Univ São Paulo. 2016 jan./abr.;27(1):42-51.

24. Hinman RS, Dobson F, Takla A, O’Donnell J, Bennell $\mathrm{KL}$. Which is the most useful patient-reported outcome in femoroacetabular impingement? Test-retest reliability of six questionnaires. Br J Sports Med. 2014;48(6):458-63. doi: 10.1136/bjsports-2012-092072.

Artigo recebido em: 24.09.15

Artigo aceito em: 23.03.16
25. Schuster C, McCaskey M, Ettlin T. German translation, cross-cultural adaptation and validation of the whiplash disability questionnaire. Health Qual Life Outcomes. 2013;11:45. doi: 10.1186/1477-7525-11-45. 